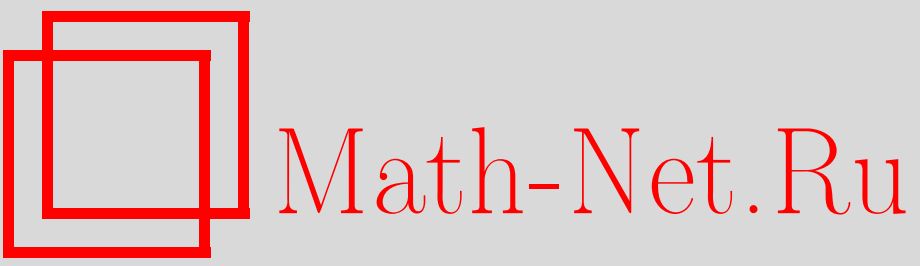

А. А. Джалилов, Сингулярные инвариантные меры гомеоморфизмов окружности с изломами, УМH, 1999, том 54, выпуск 4, 165-166

DOI: https://doi.org/10.4213/rm188

Использование Общероссийского математического портала Math-Net.Ru подразумевает, что вы прочитали и согласны с пользовательским соглашением

http://www.mathnet.ru/rus/agreement

Параметры загрузки:

IP: 54.89 .56 .158

26 апреля 2023 г., 03:33:12 


\title{
СИНГУЛЯРНЫЕ ИНВАРИАНТНЫЕ МЕРЫ ГОМЕОМОРФИЗМОВ ОКРУЖКНСТИ С ИЗЛОМАМИ
}

\author{
А. А. ДжАлилов
}

В настоящей работе изучаются гомеоморфизмы окружности с особенностями типа излома. Пусть $T_{f} x=\{f(x)\}, x \in S^{1}=[0,1)$, где $\{\cdot\}$ обозначает дробную часть числа, а функция $f(x)$ удовлетворяет следующим условиям:

a) $f(x)$ - непрерывная строго возрастающая функция и $0<f(0)<1$;

b) $f(x+1)=f(x)+1$ для любого $x \in \mathbb{R}^{1}$;

c) $f \in C^{2+\varepsilon}\left(S^{1} \backslash\left\{x_{0}\right\}\right), \varepsilon>0, f^{\prime}(x)>$ const $>0 \forall x \in S^{1} \backslash\left\{x_{0}\right\}$;

d) $\exists f^{\prime}\left(x_{0}-0\right), \exists f^{\prime}\left(x_{0}+0\right)$ и $f^{\prime}\left(x_{0}-0\right) / f^{\prime}\left(x_{0}+0\right)=c \neq 1$.

Обозначим через $\rho_{f}$ число врашения $T_{f}: \rho_{f}=\lim _{n \rightarrow \infty} f^{n}(x) / n$, здесь $f^{n}(x)$ означает $n$-ю итерацию функции $f$. Всюду в дальнейшем мы будем предполагать, что число вращения $\rho_{f}$ является иррациональным. Отсюда следует, что $T_{f}$ является строго эргодическим, т.е. для $T_{f}$ существует единственная инвариантная мера $\mu$. Из теоремы Данжуа [1], [2] следует, что гомоморфизм $T_{f}$ топологически сопряжен с линейным поворотом $T_{\rho}, T_{\rho} x=\{x+\rho\}$, т.е. существует сопрягающий гомеоморфизм $T_{\varphi}$ такой, что $T_{\varphi}\left(T_{f} x\right)=T_{\rho}\left(T_{\varphi} x\right) \forall x \in S^{1}$. Хорошо известно, что $T_{\varphi} x=\mu([0, x]), x \in S^{1}$. Если диффеоморфизм $T_{f} \in C^{2+\varepsilon}\left(S^{1}\right), \varepsilon>0$, то для типичных чисел вращения $T_{\varphi} \in C^{1+\delta}\left(S^{1}\right), 0<\delta<\varepsilon$ [3]. Отсюда следует, что инвариантная мера $\mu$ является абсолютно непрерывной относительно меры Лебега $\lambda$. Если гомеоморфизм имеет точку излома, результат будет диаметрально противоположным. А именно, если поднятие $f(x)$ гомеоморфизма $T_{f}$ удовлетворяет условиям a)-d) и число вращения иррационально, то инвариантная мера $\mu$ является сингулярной относительно меры Лебега $\lambda$ (см. [4]).

Обозначим $\underline{\tau}(x)=\underline{\lim }_{\varepsilon \rightarrow 0} \ln \mu([x, x+\varepsilon]) / \ln |\varepsilon|, \bar{\tau}(x)=\varlimsup_{\varepsilon \rightarrow 0} \ln \mu([x, x+\varepsilon]) / \ln |\varepsilon|$.

ОПРЕДЕЛЕниЕ 1 . Функции $\bar{\tau}(x)$ и $\underline{\tau}(x)$ называются соответственно верхним и нижним показателем сингулярности для меры $\mu$.

Из инвариантности функций $\underline{\tau}(x), \bar{\tau}(x)$ относительно $T_{f}$ и эргодичности меры $\mu$ следует, что они являются постоянными на множестве полной $\mu$-меры. Эти постоянные обозначим через $\underline{\tau}(\mu)$ и $\bar{\tau}(\mu)$ соответственно. Используя соображения, аналогичные тем, что были использованы в [1] для диффеоморфизмов, нетрудно показать, что гомеоморфизмы рассматриваемого нами класса являются эргодическими относительно меры Лебега. Отсюда следует, что $\underline{\tau}(x)$ и $\bar{\tau}(x)$ - почти постоянные функции по мере Лебега, обозначим их через $\underline{\tau}(\lambda)$ и $\bar{\tau}(\lambda)$.

Теорема 1. Пусть поднятие $f(x)$ гомеоморфизма $T_{f}$ удовлетворяет условиям а) -d), число вращения $\rho(f)$ иррационально и коэффициенты разложения $\rho(f)$ в непрерывную дробь ограничены. Тогда справедливы следующие неравенства: а) $0<\underline{\tau}(\mu) \leqslant \bar{\tau}(\mu)<1$; b) $1<\underline{\tau}(\lambda) \leqslant \bar{\tau}(\lambda)<\infty$.

Аналогичная теорема для критических гомеоморфизмов из класса $C^{3}$ была доказана в [5].

Приведем пример, когда нижний и верхний показатели сингулярности совпадают. Рассмотрим множество $X$ непрерывных, строго возрастающих пар $(f(x), x \in[-1,0] ; g(x), x \in[0, \alpha])$, удовлетворяюших следуюшим условиям:

1) $f(0)=\alpha, \alpha>0, g(0)=-1, f(\alpha)=g(1)$; 2) $f(-1)<0, f^{(2)}(-1)>0$.

При помощи $(f, g)$ из $X$ можно построить гомеоморфизм окружности: $T x=f(x)$ при $-1 \leqslant x<0 ; T x=g(x)$ при $0 \leqslant x<\alpha$. Обозначим через $X_{0}$ подмножество $X$, состоящее из таких пар $(f, g)$, что число врашения соответствуюших гомеоморфизмов окружности равно “золотому сечению", т.е. $\rho_{0}=(\sqrt{5}-1) / 2$. На $X_{0}$ определим преобразование ренормгруппы: $R_{1}(f(x),-1 \leqslant x<0 ; g(x), 0 \leqslant x<\alpha)=\left(f^{\prime}(x),-1 \leqslant x<0 ; g^{\prime}(x), 0 \leqslant x<\alpha^{\prime}\right)$, где $f^{\prime}(x)=-\alpha^{-1} f(g(-\alpha x)), g^{\prime}(x)=-\alpha^{-1} f(-\alpha x), \alpha^{\prime}=-\alpha^{-1} f(-1)$.

Обозначим $c=f^{\prime}(-0) / f^{\prime}(+0)$. В работе [2] доказано, что при фиксированном $c, c \neq 1$, преобразование $R_{1}$ в $X_{0}$ имеет единственную периодическую траекторию $\left\{\left(f_{i}, g_{i}\right), i=1,2\right\}$ периода 2. Функции $f_{i}(x), g_{i}(x), i=1,2$, являются дробно-линейными функциями. Обозначим через 
$T_{1}$ гомеоморфизм окружности $\left[-1, \alpha_{1}\right)$, отвечаюший $\left(f_{1}, g_{1}\right)$ (пара $\left(f_{2}, g_{2}\right)$ изучается аналогичным образом). Ясно, что $\rho\left(T_{1}\right)=\rho_{0}$. Хорошо известно, что разложение $\rho_{0}$ в непрерывную дробь имеет вид $\rho_{0}=[1,1, \ldots, 1, \ldots]$. Обозначим $p_{n} / q_{n} n$-ю подходящую дробь $\rho_{0}$. Числа $q_{n}$ удовлетворяют следующему рекуррентному соотношению: $q_{n+1}=q_{n}+q_{n-1}, n \geqslant 1, q_{0}=q_{1}=1$. Обозначим $x_{i}=T_{1}^{i} x_{0}, i \geqslant 1, x_{0}=0$, и $\Delta_{0}^{(n)}$ - замкнутый отрезок, соединяющий точки $x_{0}$ и $x_{q_{n}}$. Положим $\Delta_{i}^{(n)}=T_{1}^{i} x_{0}, i \geqslant 1$.

Лемма (см. [6]). Для каждого $n$ системы отрезков $\Delta_{i}^{(n-1)}, 0 \leqslant i<q_{n} ; \Delta_{j}^{(n)}, 0 \leqslant$ $j<q_{n-1}$, не пересекаются (за исключением концевых точек) и образуют разбиение окружсности.

Полученное разбиение обозначим $\xi_{n-1}$. При переходе от $\xi_{n-1}$ к $\xi_{n}$ происходит следующее. Все отрезки $\Delta_{j}^{(n)}, 0 \leqslant j<q_{n-1}$, сохраняются, а каждый из отрезков $\Delta_{i}^{(n-1)}, 0 \leqslant i<q_{n}$, распадается на два отрезка: $\Delta_{i}^{(n-1)}=\Delta_{i}^{(n+1)} \cup \Delta_{i+q_{n-1}}^{(n)}$.

Теперь, используя последовательность разбиений, построим символическое представление для всех точек $x \in S \backslash\left\{x_{i}, i \geqslant 0\right\}$. Положим $a_{n}=a$, если $x \in \Delta_{i}^{(n)}, 0 \leqslant i<q_{n}$, и $a_{n}=1$, если $x \in \Delta_{i+q_{n-1}}^{(n)}$. Если $x \in \Delta_{i}^{(n+1)}$, то $a_{n}=0$. Таким образом, мы получили взаимно однозначное соответствие: $x \leftrightarrow\left(a_{0}, a_{1}, \ldots, a_{n}, \ldots\right)=\vec{a}$, где $a_{0}=a, 0 ; a_{n}=a, 0,1, n \geqslant 1$; (легко видеть, что $a_{n}=a$, если и только если $\left.a_{n-1}=0\right)$. Множество всех таких слов обозначим $A$.

Теорема 2. Существует функция $U: A \rightarrow R_{1}$, обладающая следующими свойствамu:

1) функция $U(\vec{a})$ является непрерывной (в тихоновской топологии), и существуют константы $c_{i}<0, i=1,2$, такие, что для любого $\vec{a} \in A \quad c_{1} \leqslant U(\vec{a}) \leqslant c_{2}$;

2) пусть $\vec{a}, \vec{b} \in A$ и $a_{i}=b_{i}, 0 \leqslant i \leqslant k$, тогда $|U(\vec{a})-U(\vec{b})| \leqslant$ const $q^{k}$, где константа $q, 0<q<1$, не зависит от $k, \vec{a} u \vec{b}$

3) пусть $\Delta_{s}^{(n)} \subset \Delta_{m}^{(n-k)}$ и әтим отрезкам соответствуют слова $\left(a_{0}, a_{1}, \ldots, a_{k}\right.$, $\left.a_{k+1}, \ldots, a_{n-1}\right) u\left(a_{0}, a_{1}, \ldots, a_{n-k-1}\right)$, тогда

$$
\left|\Delta_{m}^{(n)}\right|=\left|\Delta_{m}^{(n-k)}\right| \exp \left\{\sum_{s=n-k}^{n-1} U\left(a_{s}, a_{s-1}, \ldots, a_{0}, \vec{\nu}\right)\right\}(1+\psi(\vec{a})),
$$

где $\vec{\nu}$ - такой вектор, что $\left(a_{0}, \vec{\nu}\right) \in A, u|\psi(\vec{a})| \leqslant \operatorname{const} q^{k}$.

Пусть $x \in\left[-1, \alpha_{1}\right)$ и $\Delta^{(n)}(x)$ - отрезок разбиения $\xi_{n}$, содержащий $x$. Обозначим $\Delta_{n}=$ $\left|q_{n} \rho-p_{n}\right|$, т.е. $\Delta_{n}-$ длина отрезка $n$-го ранга при линейном повороте на угол $\rho$.

ТеОрема 3. Для почти всех $x$ по мере Лебега (по мере $\mu$ ) существует конечный предел $\lim _{n \rightarrow \infty} \ln \Delta_{n} / \ln \left|\Delta^{(n)}(x)\right|=\gamma(\lambda) \quad(\gamma(\mu)) u \underline{\tau}(\lambda)=\bar{\tau}(\lambda)=\gamma(\lambda), \underline{\tau}(\mu)=\bar{\tau}(\mu)=\gamma(\mu)$.

\section{СПИСОК ЛИТЕРАТУРЫ}

[1] Корнфельд И. П., Синай Я.Г., Фомин С. В. Эргодическая теория. М.: Наука, 1980. [2] Khanin K. M., Vul E. B. // Adv. Soviet Math. 1991. V. 3. P. 57-98. [3] Синай Я. Г., Ханин К. М. // УМН. 1989. Т. 44. №1. С. 57-82. [4] Джалилов А. А., Ханин К. М. // Функц. анализ и его прил. 1998. Т. 32. № 3. С. 11-21. [5] Graczyk J., Swiatek G. // Comm. Math. Phys. 1993. V. 157. № 2. P. 213-230. [6] Синай Я. Г. Современные проблемы эргодической теории. М.: Физматлит, 1995. 